\title{
Identifying systematic reviews of the adverse effects of health care interventions
}

\author{
Su Golder*1, Heather M McIntosh ${ }^{1,2}$ and Yoon Loke ${ }^{3}$
}

\author{
Address: ${ }^{1}$ Centre for Reviews and Dissemination (CRD), University of York, York, YO10 5DD, UK, ${ }^{2}$ NHS Quality Improvement Scotland, Delta \\ House, 50 West Nile St, Glasgow, G1 2NP, UK and ${ }^{3}$ University of East Anglia Norwich NR4 7TJ, UK \\ Email: Su Golder* -spg3@york.ac.uk; Heather M McIntosh - heather.mcintosh@nhshealthquality.org; Yoon Loke - Y.Loke@uea.ac.uk \\ * Corresponding author
}

Published: 08 May 2006

BMC Medical Research Methodology 2006, 6:22 doi:10.1 186/147I-2288-6-22

This article is available from: http://www.biomedcentral.com/I47I-2288/6/22

(c) 2006 Golder et al; licensee BioMed Central Ltd.

This is an Open Access article distributed under the terms of the Creative Commons Attribution License (http://creativecommons.org/licenses/by/2.0), which permits unrestricted use, distribution, and reproduction in any medium, provided the original work is properly cited.
Received: 18 October 2005

Accepted: 08 May 2006

\begin{abstract}
Background: In order to carry out a methodological research survey of systematic reviews of adverse effects we needed to retrieve a sample of systematic reviews in which the primary outcome is an adverse effect or effects.

Methods: We carried out searches of the Database of Abstracts of Reviews of Effects (DARE) and the Cochrane Database of Systematic Reviews (CDSR) for systematic reviews of adverse effects published between 1994 to 2005. The search strategies used a combination of text words in the title and abstract, Medical Subject Headings (MeSH) and subheadings/qualifiers. In addition, DARE records in progress were hand searched. No language restrictions were placed on any of the searches. The performance, in terms of sensitivity and precision, of the search strategies and their combinations were tested in DARE and CDSR.
\end{abstract}

Results: In total 3635 records were screened of which 257 met our inclusion criteria. The precision of the searches in CDSR was low ( $0 \%$ to $3 \%$ ), and no one search strategy could retrieve all the relevant records in either DARE or CDSR. Hand searching the records from DARE and CDSR not retrieved by our searches indicated that we had missed relevant systematic reviews in both DARE and CDSR. The sensitivities of many of the search combinations were comparable to those found when searching for primary studies in which adverse effects are secondary outcomes.

Conclusion: Searching major databases of systematic reviews, for systematic reviews of adverse effects, proved more difficult than anticipated due to a lack of standard terminology used by the authors, inadequate indexing and the variations in the search interfaces of the databases. At present hand searching all records in DARE and CDSR seems to be the only way to ensure retrieval of all systematic reviews of adverse effects in these databases.

\section{Background}

Balanced decision making in health care requires evidence on the potential adverse effects of interventions as well as their beneficial effects. Although well-conducted systematic reviews of adverse effects are important sources of evi- dence such reviews are relatively rare in the literature [1] and it is not clear whether the process of identifying relevant reviews may resemble the proverbial "looking for a needle in the haystack". Indeed, poor indexing and inconsistent terminology have hampered efforts to identify 
studies that report original data on adverse effects [2-5]. These primary studies often do not consider adverse effects as the main outcome and, therefore, may not contain this information in their title or abstract or be indexed with terms for adverse effects.

It should be easier to identify systematic reviews that were conducted with the express purpose of evaluating adverse effects. We might expect that study retrieval would be facilitated by some mention of adverse effects in the title, abstract or indexing terms. As part of a wider study of methods used in systematic reviews of adverse effects we decided to assess whether we could identify systematic reviews of adverse effects quickly and easily in two major databases of systematic reviews.

\section{Methods}

We searched for systematic reviews of adverse effects using the Database of Abstracts of Reviews of Effects (DARE) and the Cochrane Database of Systematic Reviews (CDSR). These databases were chosen because they are major collections of systematic reviews. No additional sources were searched as DARE is compiled through rigorous monthly searches of bibliographic databases (including MEDLINE and EMBASE) as well as hand searching key journals, grey literature, and regular searches of the web [6]. No language restrictions were placed on the searches and the searches aimed to retrieve systematic reviews published from 1994 onwards.

\section{Database of Abstracts of Reviews of Effects (DARE)}

Three approaches were used to identify records in DARE (figure 1). Firstly, text word searches for synonyms of 'adverse effects' and related terms were carried out in the record title and abstract. These terms were selected from previous research [4]. Each DARE abstract contains a summary of a systematic review including a critical commentary. It was found that searching the full abstracts of DARE records would have identified many irrelevant records as these abstracts contain phrases such as; 'no information on the incidence of adverse reactions are included', 'it would have been appropriate to include mention of adverse events' or 'the adverse effects of the treatment were not assessed in the review'. The searches of the DARE abstracts were, therefore, restricted to the 'outcomes assessed in the review' field as the primary outcome of a review is described in this field [7].

The second approach was to use Medical Subject Headings (MeSH), such as DRUG TOXICITY, and subheadings/ qualifiers unattached to any indexing terms ('floating' subheadings), such as 'adverse effects'. This was an essential part of the search strategy as many systematic reviews examine specific adverse effects, such as, headaches, so would not necessarily be identified by text words of syno- nyms of 'adverse effects' and searching for each named potential adverse effect individually would be impractical. Previous research has also indicated the usefulness of searching with 'floating' adverse effect subheadings [2-4].

Finally, DARE records in the process of being written do not yet have an 'outcomes assessed in the review' field. The titles of all of these 'provisional' records were, therefore, hand searched by the researchers to identify additional relevant reviews.

To enable all three approaches to be executed, three searches of DARE were conducted, two via the Centre for Reviews and Dissemination (CRD) website and one via The Cochrane Library website (figure 1). The text word search was conducted using the CRD website because this interface allows searches to be limited to sections of the structured abstracts, such as, the 'outcomes assessed in the review' field, whereas The Cochrane Library interface does not. The provisional abstracts were scanned via the CRD website as this contains the most up to date set of provisional DARE records. Another search was conducted using The Cochrane Library because its interface allows searches of 'floating' subheadings to be conducted whereas the CRD website does not.

\section{The Cochrane Database of Systematic Reviews (CDSR)}

Searches for Cochrane Reviews were conducted in the web version of The Cochrane Library (Issue 1: 2005) (figure 2). These searches used text words in the title and abstract, MeSH terms, and 'floating' subheadings. As with DARE records many irrelevant records would have been retrieved if the full CDSR structured abstracts had been searched. The 'objectives' section of a CDSR abstract outlines the primary outcome of a review [8]. As searches in CDSR cannot be limited to sections of the abstract text words were searched for in the abstract using the proximity operator to limit to within 20 words of the term 'objectives' (figure 2).

\section{Inclusion criteria}

The results from all four searches were then entered into an Endnote Library and duplicate records were removed. Two researchers independently screened the titles and abstracts and selected records for inclusion in the study. A review was included if the primary outcome was an adverse effect or effects, that were known to be, or suspected of being, associated with the intervention. This was regardless of whether the review indicated that the intervention increased or reduced the outcome.

It was suspected that relevant reviews had been missed by the searches so those records not retrieved by the search strategies in CDSR $(\mathrm{n}=887)$ and DARE $(\mathrm{n}=2646)$ were also scanned for relevant systematic reviews. All relevant 
Search Approach 1: Searching with text words in the title and abstract DARE searched via CRD website at http://www.york.ac.uk/inst/crd/ Searched: $31 / 03 / 05$

(safe or safety or adverse or tolerability or toxicity or toxic or adrs or adr or tolerance or tolerate or harm or harms or harmful or complication\$ or risk or risks)/xoa OR side(w)effect\$/xoa OR undesirable(w)effect\$/xoa OR treatment(w)emergent/xoa OR (safe or safety or adverse or tolerability or toxicity or toxic or adrs or adr or tolerance or tolerate or harm or harms or harmful or complication $\$$ or risk or risks)/ttl OR side(w)effect\$/ttl OR undesirable(w)effect\$/ttl OR treatment(w)emergent/ttl

\section{Key}

/xoa - restricts the search to the 'outcomes assessed in the review' field

/ttl - restricts the search to the 'title' field

$\$$ - truncation symbol

(w) - words must be adjacent to each other

Search Approach 2: Searching using indexing terms

DARE searched via the Cochrane Library at http://www.nelh.nhs.uk/cochrane.asp

Issue 12005

Searched: 31/03/05

\#1 Any MeSH descriptor with qualifier: AE \#2 Any MeSH descriptor with qualifier: DE \#3 Any MeSH descriptor with qualifier: $\mathrm{CO}$ \#4 Any MeSH descriptor with qualifier: PO \#5 Any MeSH descriptor with qualifier: TO \#6 Any MeSH descriptor with qualifier: CI \#7 MeSH descriptor Drug Hypersensitivity explode all trees \#8 MeSH descriptor Drug Toxicity explode all trees \#9 MeSH descriptor Product Surveillance, Postmarketing explode all trees $\# 10 \# 1$ or \#2 or \#3 or \#4 or \#5 or \#6 or \#7 or \#8 or \#9

\section{Key}

Sets \#1 to \#6 are 'floating' subheadings

\section{Search Approach 3: Searching 'provisional' abstracts}

All records in progress, 'provisional' abstracts, were scanned for relevant reviews of adverse effects.

Figure I

Search strategies for retrieving systematic reviews of adverse effects from DARE. 


\section{Cochrane Database of Systematic Reviews (CDSR) searched via The Cochrane Library at http://www.nelh.nhs.uk/cochrane.asp}

Issue 12005

Searched: 31/03/05

\#1 Any MeSH descriptor with qualifier: AE \#2 Any MeSH descriptor with qualifier: DE \#3 Any MeSH descriptor with qualifier: $\mathrm{CO}$ \#4 Any MeSH descriptor with qualifier: PO \#5 Any MeSH descriptor with qualifier: TO \#6 Any MeSH descriptor with qualifier: CI \#7 MeSH descriptor Drug Hypersensitivity explode all trees \#8 MeSH descriptor Drug Toxicity explode all trees \#9 MeSH descriptor Product Surveillance, Postmarketing explode all trees \#10 (safe or safety or adverse or tolerability or toxicity or toxic or adrs or adr or tolerance or tolerate or harm or harms or harmful or complication* or risk or risks) near/20 objective* in Abstract \#11 (side next effect*) near/20 objective* in Abstract \#12 (undesirable next effect*) near/20 objective* in Abstract \#13 (treatment next emergent) near/20 objective* in Abstract \#14 (safe or safety or adverse or tolerability or toxicity or toxic or adrs or adr or tolerance or tolerate or harm or harms or harmful or complication* or risk or risks) in Record Title \#15 (side next effect*) in Record Title \#16 (undesirable next effect*) in Record Title \#17 (treatment next emergent) in Record Title $\# 18 \# 1$ or \#2 or \#3 or \#4 or \#5 or \#6 or \#7 or \#8 or \#9 or \#10 or \#11 or \#12 or \#13 or $\# 14$ or \#15 or \#16 or \#17

\section{Key}

Sets \#1 to \#6 are 'floating' subheadings next - words must be adjacent to each other near/20 - words must be within 20 words of each other in either direction

\section{Figure 2}

Search strategies for retrieving systematic reviews of adverse effects from CDSR. 

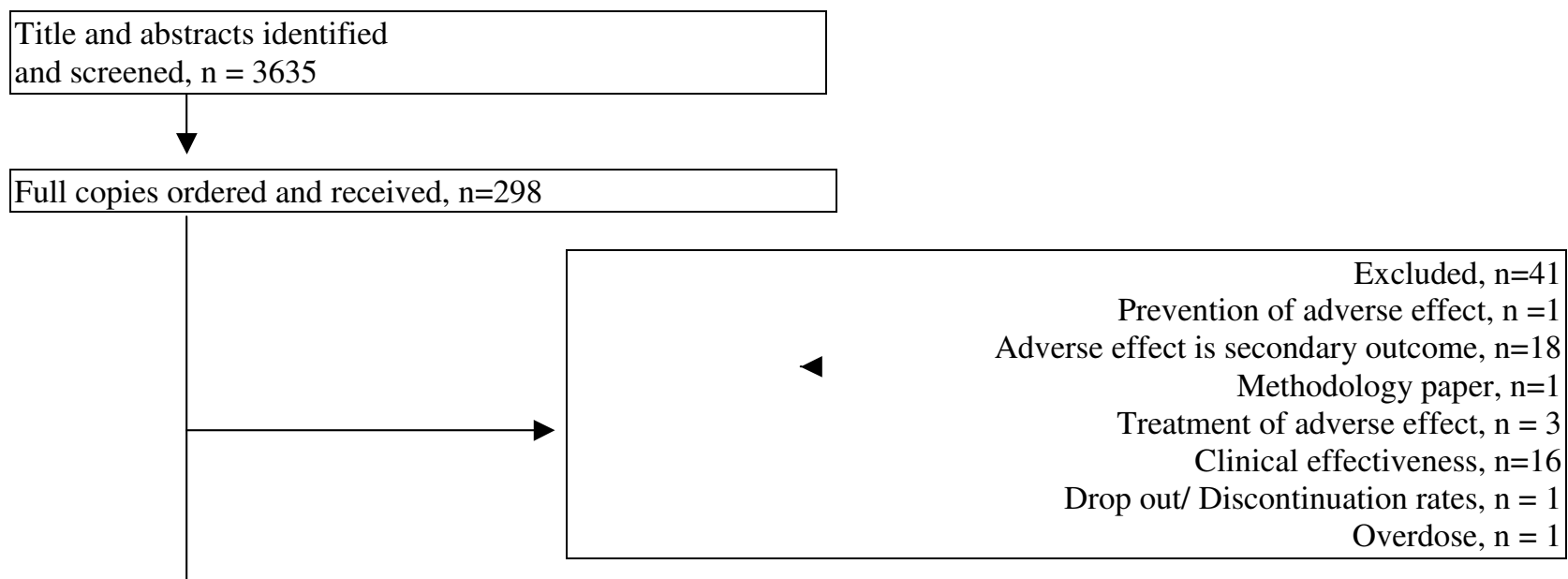

Publications meeting the inclusion criteria

and included in the review, $\mathrm{n}=257$

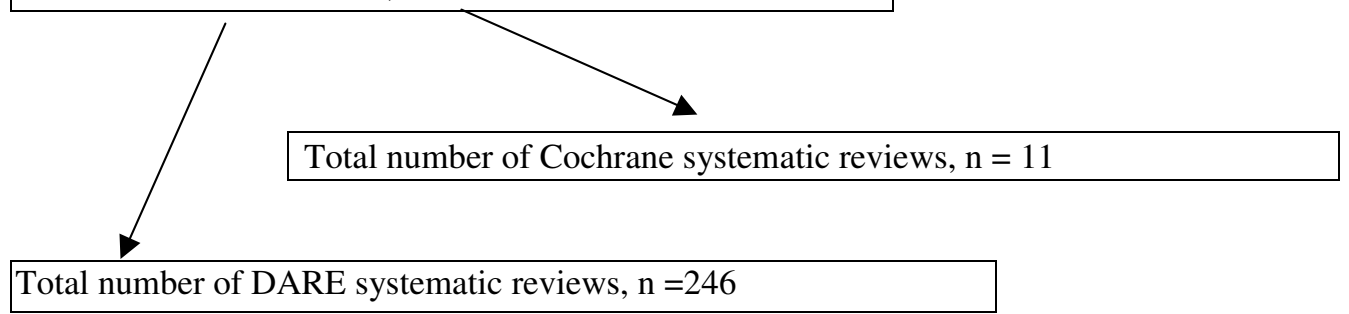

Figure 3

Summary of systematic review identification, retrieval and inclusion/exclusion.

records identified then formed our gold standard (GS) set of records.

\section{Assessing the performance of the search strategies}

Once we had established our gold standard set of records we were able to test the performance of individual approaches in retrieving the gold standard records. The search terms used to identify the systematic reviews were assessed for their usefulness in retrieving relevant records by measuring their sensitivity and precision. Sensitivity is a measure of the search's ability to identify relevant papers, and a high value is important for searches for systematic reviews. Precision, on the other hand, is a measure of the proportion of relevant records identified by a search strategy expressed as a percentage of all articles (relevant and irrelevant) identified by that strategy. Highly sensitive strategies tend to have low levels of precision. Sensitivity and precision for each database were calculated as follows; $\begin{aligned} \text { Sensitivity }= & \frac{\text { number of GS records retrieved }}{\text { number of GS records indexed }} \\ & \text { in the database under investigation }\end{aligned}$

Precision $=\frac{\text { number of GS records retrieved }}{\text { total number of records retrieved }} \times 100$

\section{Results}

In total 4262 records were retrieved from CDSR and DARE, of which 3635 were unique records. From the 3635 titles and abstracts screened, 298 full reports were retrieved and 256 reviews (257 publications) met our inclusion criteria. Of the 257 publications, 246 had DARE abstracts and 11 were Cochrane Reviews (figure 3 ).

The hand search of the records in CDSR and DARE not retrieved by our search strategies identified 13 additional records ( 10 from DARE and 3 from CDSR) which met our inclusion criteria. In total, therefore, 270 systematic 
Table I: Sensitivity and precision of searches in DARE and CDSR

\begin{tabular}{|c|c|c|c|c|}
\hline & No of Papers Retrieved & No of Relevant Papers & Sensitivity (\%) & Precision (\%) \\
\hline \multicolumn{5}{|l|}{ DARE (Quasi Gold Standard = 256) } \\
\hline $\begin{array}{l}\text { 'floating' subheadings: 'adverse effects' OR 'drug effects' OR } \\
\text { 'complications' OR 'poisoning' OR 'toxicity' OR 'chemically } \\
\text { induced' }\end{array}$ & 1386 & 217 & $85 \%$ & $16 \%$ \\
\hline $\begin{array}{l}\text { 'floating' subheadings: 'adverse effects' OR 'drug effects' OR } \\
\text { 'complications' OR 'poisoning', 'toxicity' OR 'chemically } \\
\text { induced' OR Exp DRUG HYPERSENSITIVITY OR Exp DRUG } \\
\text { TOXICITY OR EXP PRODUCT SURVEILLANCE, } \\
\text { POSTMARKETING }\end{array}$ & 1386 & 217 & $85 \%$ & $16 \%$ \\
\hline $\begin{array}{l}\text { synonyms of 'adverse effects' in the 'title' OR 'outcomes } \\
\text { assessed in the review' field }\end{array}$ & 873 & 160 & $63 \%$ & $18 \%$ \\
\hline synonyms of 'adverse effects' in the title & 462 & 132 & $52 \%$ & $29 \%$ \\
\hline $\begin{array}{l}\text { synonyms of 'adverse effects' in 'outcomes assessed in the } \\
\text { review' field }\end{array}$ & 659 & 116 & $45 \%$ & $18 \%$ \\
\hline $\begin{array}{l}\text { exp DRUG HYPERSENSITIVITY OR exp DRUG TOXICITY } \\
\text { OR exp PRODUCT SURVEILLANCE, POSTMARKETING }\end{array}$ & 14 & 10 & $4 \%$ & $71 \%$ \\
\hline \multicolumn{5}{|l|}{ CDSR (Quasi Gold Standard = 14) } \\
\hline $\begin{array}{l}\text { 'floating' subheadings: 'adverse effects' OR 'drug effects' OR } \\
\text { 'complications' OR 'poisoning', 'toxicity' OR 'chemically } \\
\text { induced' }\end{array}$ & 416 & 9 & $64 \%$ & $3 \%$ \\
\hline $\begin{array}{l}\text { synonyms of 'adverse effects' near/20 objectives in the 'abstract' } \\
\text { field }\end{array}$ & 1049 & 5 & $36 \%$ & $0.5 \%$ \\
\hline synonyms of 'adverse effects' in the 'title' field & 64 & 2 & $14 \%$ & $3 \%$ \\
\hline $\begin{array}{l}\text { exp DRUG HYPERSENSITIVITY OR exp DRUG TOXICITY } \\
\text { OR exp PRODUCT SURVEILLANCE, POSTMARKETING }\end{array}$ & 2 & 0 & $0 \%$ & $0 \%$ \\
\hline
\end{tabular}

reviews of adverse effects were identified; 256 from DARE and 14 from CDSR.

The relevant records not retrieved by our search strategies were sifted for any potentially relevant generic adverse effect search terms. Only 2 of the 13 contained potentially useful terms. Both contained the MeSH indexing term RISK FACTORS and one had the term 'hazards' in the title. These search terms, in addition to the terms used in our search strategies, were tested to identify the most sensitive search strategy possible.

The sensitivity and precision of the different search approaches are presented in table 1. Searching using 'floating' subheadings provided the highest sensitivity in both DARE (85\%) and CDSR (64\%) and the precision of all the search approaches was much higher in DARE (16\% to $71 \%$ ) than in CDSR ( $0 \%$ to $3 \%$ ).

\section{Single search terms with the highest precision}

All the single search terms in CDSR yielded very low precision (0 to 3\%). In DARE, however, some terms did provide high precision (table 2 ). The single terms with the highest precision were the MeSH terms, Exp PRODUCT SURVELLIENCE, POSTMARKETING (73\%), Exp DRUG HYPERSENSITIVITY (67\%) and Exp DRUG TOXICITY (67\%). However, the sensitivity of searching with each of these terms was very low (1-3\%) (table 2). Searches using all the chosen synonyms of 'adverse effects' in the title had a reasonable precision of $29 \%$ (table 1 ). However, some individual single terms yielded higher precision. For example, searching with the term 'adverse' in the title gave $65 \%$ precision, 'complication\$' $50 \%$ and 'side effect\$' $43 \%$.

\section{Single search terms with the highest sensitivity}

The most sensitive search strategy used 'floating' subheadings (table 1). 'Floating' all the subheadings retrieved $85 \%$ of the DARE records and $64 \%$ of the Cochrane Reviews. The most sensitive 'floating' subheadings in DARE were 'adverse-effects' at $77 \%$, followed by 'chemically-induced' (33\%), 'drug-effects' (16\%), and 'complications' (14\%) (table 3). In CDSR 'adverse-effects' was again the most sensitive 'floating' subheading (64\%), followed by 'chemically-induced' (29\%), and 'drug-effects' (29\%).

\section{Most sensitive search strategies}

The most sensitive search strategy in DARE, with the terms tested here, used a combination of text words in the title and abstract, a MeSH term and 'floating' subheadings (see figure 4). This strategy retrieved 1,507 records of which 241 were deemed relevant, yielding a sensitivity of $94 \%$ and precision of $16 \%$.

In CDSR the most sensitive search strategy used the 'floating' subheading 'adverse effects' combined with searching 
Table 2: Single search terms with highest precision in DARE (Quasi Gold Standard $=\mathbf{2 5 6}$ )

\begin{tabular}{|c|c|c|c|c|c|}
\hline Search Term & Field Searched & No of Papers Retrieved & No of Relevant Papers & Precision (\%) & Sensitivity (\%) \\
\hline exp DRUG HYPERSENSITIVITY & indexing & II & 8 & $73 \%$ & $3 \%$ \\
\hline $\exp$ DRUG TOXICITY & indexing & 3 & 2 & $67 \%$ & $1 \%$ \\
\hline $\begin{array}{l}\text { exp PRODUCT SURVEILLANCE, } \\
\text { POSTMARKETING }\end{array}$ & indexing & 3 & 2 & $67 \%$ & $1 \%$ \\
\hline adverse & title & 40 & 26 & $65 \%$ & $10 \%$ \\
\hline complication\$ & title & 28 & 14 & $50 \%$ & $5 \%$ \\
\hline side effect $\$$ & title & 7 & 3 & $43 \%$ & $1 \%$ \\
\hline 'toxicity' (floating subheading) & indexing & 7 & 3 & $43 \%$ & $1 \%$ \\
\hline 'chemically-induced' (floating subheading) & indexing & 201 & 85 & $42 \%$ & $33 \%$ \\
\hline adverse & outcomes assessed & 78 & 32 & $41 \%$ & $13 \%$ \\
\hline risk & title & 140 & 55 & $39 \%$ & $21 \%$ \\
\hline side effect $\$$ & outcomes assessed & 3 & $\mathrm{l}$ & $33 \%$ & $0 \%$ \\
\hline risk & outcomes assessed & 168 & 54 & $32 \%$ & $21 \%$ \\
\hline safety & title & 96 & 29 & $30 \%$ & $11 \%$ \\
\hline complication\$ & outcomes assessed & 46 & 13 & $28 \%$ & $5 \%$ \\
\hline risks & outcomes assessed & 39 & 10 & $26 \%$ & $4 \%$ \\
\hline
\end{tabular}

for 'adverse near/20 objectives' in the abstract. This strategy retrieved $79 \%(11 / 14)$ of the relevant records. However, the precision of this search was low at $3 \%(11 / 338)$.

\section{Discussion}

This research highlights the advantages and disadvantages of searching databases through the CRD website and The Cochrane Library website. The CRD website offered the most current version of DARE and allowed searches to be limited to sections of the abstract, whereas The Cochrane Library version of DARE allowed searching using 'floating' subheadings. Even when conducting consecutive searches on DARE and CDSR in two different interfaces it is difficult to retrieve all systematic reviews of adverse effects on these databases. A sensitive search using text words in the title and abstract, indexing terms and 'floating' subheadings was unable to retrieve all the records of interest. An assessment of the missed systematic reviews indicated that most of these records could not have been retrieved without searching for specific adverse effects. Although adding these terms to our search strategy would have increased the sensitivity of the searches, adding the MeSH term RISK FACTORS, in particular, would have decreased the precision.

Research has indicated that primary studies of adverse effects are difficult to locate [2-5]. This has been attributed to poor reporting, inconsistent terminology and inadequate indexing. In primary studies adverse effects are often not the main outcome of the study and are described secondary to clinical effectiveness. In this case study we sought systematic reviews in which the primary outcome was an adverse effect or effects. It was anticipated that such studies would be easier to retrieve because adverse effects would more likely be contained in their title and abstract and thus their indexing. This was tested by comparing the sensitivities and precision of our searches to those reported in earlier research on primary studies.

Table 3: Single search terms with the highest sensitivity in DARE (Quasi Gold Standard = 256)

\begin{tabular}{|c|c|c|c|c|c|}
\hline Search Term & Field Searched & No of Papers Retrieved & No of Relevant Papers & Sensitivity (\%) & Precision (\%) \\
\hline 'adverse-effects' ('floating' subheading) & indexing & 898 & 198 & $77 \%$ & $22 \%$ \\
\hline $\begin{array}{l}\text { 'chemically-induced' ('floating' } \\
\text { subheading) }\end{array}$ & indexing & 201 & 85 & $33 \%$ & $42 \%$ \\
\hline RISK FACTORS & indexing & 336 & 69 & $27 \%$ & $21 \%$ \\
\hline risk & title & 140 & 55 & $21 \%$ & $39 \%$ \\
\hline risk & outcomes Assessed & 168 & 54 & $21 \%$ & $32 \%$ \\
\hline 'drug-effects' (floating subheading) & indexing & 273 & 42 & $16 \%$ & $15 \%$ \\
\hline 'complications' (floating subheading) & indexing & 550 & 36 & $14 \%$ & $7 \%$ \\
\hline adverse & outcomes assessed & 78 & 32 & $13 \%$ & $41 \%$ \\
\hline safety & title & 96 & 29 & $11 \%$ & $30 \%$ \\
\hline adverse & title & 40 & 26 & $10 \%$ & $65 \%$ \\
\hline
\end{tabular}


This requires a combination of a search in DARE in both the Cochrane Library and the CRD website;

\section{DARE searched via the Cochrane Library at http://www.nelh.nhs.uk/cochrane.asp}

\#1 Any MeSH descriptor with qualifier: AE

\#2 Any MeSH descriptor with qualifier: DE

\#3 Any MeSH descriptor with qualifier: CI

\#4 (risk or side effect or complication* or harm or tolerability or safety) in record title

\#5 MeSH descriptor Risk Factors

\#6 \#1 or \#2 or \#3 or \#4 or \#5

OR

DARE searched via CRD website at http://www.york.ac.uk/inst/crd/ risk/xoa

\section{Key}

Sets \#1 to \#3 are 'floating' subheadings

* - truncation symbol

/xoa - restricts the search to the 'outcomes assessed in the review' field

Sets \#1 to \#3 can only be searched in the Cochrane Library interface

Sets \#4 and \#5 can be searched in either the Cochrane Library interface or the CRD

website

Set 7 can only be searched via the CRD website

\section{Figure 4}

Most sensitive search strategy to retrieve adverse effects in DARE.

Interestingly our searches shared similar sensitivities to those reported by Badgett et al's [2] and Golder et al's [4] when searching with subheadings and indexing terms (table 4). The single most sensitive term in all three studies was the 'floating' subheading 'adverse effects' (table 4). It is difficult to compare the sensitivities of searching in the abstract as in this study the searches were limited to particular sections of the structured abstracts in DARE and CDSR. Searching the title for synonyms of 'adverse effects' and related terms showed a higher sensitivity in DARE in this study than in Badgett et al's [2] and Golder et al's [4]. This may reflect the fact that the relevant studies here contained an adverse effect or effects as their primary outcome. The lower sensitivity we experienced in CDSR, however, may reflect the prescribed format of Cochrane Review titles which focus on the intervention and condition [8].

Derry et al [3] found that of a sample of randomised controlled trials (RCTs) reporting adverse effects, only $77 \%$
(82/107) could be identified by adverse effects indexing terms or text words in the title or abstract. In our study, $79 \%$ (11/14) of the Cochrane Reviews and 91\% (233/ 256) of the DARE reviews could be identified by adverse effects indexing terms or text words in the title or abstract similar to those used by Derry et al [3]. On further inspection of the results from Derry et al [3], 53\% (53/100) of their MEDLINE records and 49\% (43/88) of their EMBASE records contained an adverse effect indexing term compared to $80 \%(204 / 256)$ from DARE in our case study and $64 \%(9 / 14)$ from CDSR. This indicates that systematic reviews with a main outcome of an adverse effect may be marginally easier to retrieve than the RCTs in MEDLINE and EMBASE examined by Derry et al [3] and that this may be due in part to indexing. Indexers are instructed to index only the most important subject matter in an article [9], therefore, it may not be surprising that the RCTs in Derry et al's [3] study were not all indexed with adverse effects terms. It is surprising, however, that so many of the papers whose primary outcome was an 
Table 4: Comparison of search sensitivities in three case studies

\begin{tabular}{|c|c|c|c|c|c|}
\hline Search Terms & $\begin{array}{l}\text { Sensitivity in } \\
\text { DARE in this case } \\
\text { study }(G S=256)\end{array}$ & $\begin{array}{l}\text { Sensitivity in } \\
\text { CDSR in this case } \\
\text { study }(G S=14)\end{array}$ & $\begin{array}{l}\text { Sensitivity in MEDLINE } \\
\text { in Badgett et al's study } \\
\text { [2] (QGS = 323) }\end{array}$ & $\begin{array}{l}\text { Sensitivity in MEDLINE } \\
\text { in Golder et al's study } \\
\text { [4] (QGS = 67) }\end{array}$ & $\begin{array}{l}\text { Sensitivity in EMBASE } \\
\text { in Golder et al's study } \\
\text { [4] (QGS = 72) }\end{array}$ \\
\hline $\begin{array}{l}\text { 'adverse effects' (floating } \\
\text { subheading) }\end{array}$ & $77 \%$ & $64 \%$ & $86 \%$ & $79 \%$ & $79 \%[1]$ \\
\hline $\begin{array}{l}\text { 'complications' (floating } \\
\text { subheading) }\end{array}$ & $14 \%$ & $0 \%$ & $12 \%$ & $6 \%$ & $5 \%[I]$ \\
\hline $\begin{array}{l}\text { 'poisoning' (floating } \\
\text { subheading) }\end{array}$ & $0.4 \%$ & $0 \%$ & $10 \%$ & $0 \%$ & $n / a$ \\
\hline $\begin{array}{l}\text { 'chemically induced' (floating } \\
\text { subheading) }\end{array}$ & $33 \%$ & $29 \%$ & Not tested & $28 \%$ & $\mathrm{n} / \mathrm{a}$ \\
\hline 'toxicity' (floating subheading) & $1 \%$ & $0 \%$ & Not tested & $0 \%$ & $3 \%[1]$ \\
\hline $\begin{array}{l}\text { 'drug effects' (floating } \\
\text { subheading) }\end{array}$ & $16 \%$ & $29 \%$ & Not tested & $33 \%$ & $\mathrm{n} / \mathrm{a}$ \\
\hline $\begin{array}{l}\text { 'adverse effects' OR } \\
\text { 'complications' OR 'poisoning' } \\
\text { (floating subheadings) }\end{array}$ & $79 \%$ & $57 \%$ & $95 \%$ & $82 \%$ & $81 \%$ \\
\hline $\begin{array}{l}\text { 'adverse effects OR } \\
\text { 'complications' OR 'poisoning' } \\
\text { OR 'drug effects' OR 'toxicity' } \\
\text { OR 'chemically induced' } \\
\text { (floating subheadings) }\end{array}$ & $85 \%$ & $64 \%$ & Not tested & $88 \%$ & $81 \%[1]$ \\
\hline $\begin{array}{l}\text { 'adverse effects' OR 'drug } \\
\text { effects' OR 'complications' OR } \\
\text { 'poisoning' OR 'toxicity' OR } \\
\text { 'chemically induced' (all } \\
\text { floating subheadings) OR exp } \\
\text { DRUG HYPERSENSITIVITY } \\
\text { OR exp DRUG TOXICITY } \\
\text { OR exp PRODUCT } \\
\text { SURVEILLANCE, } \\
\text { POSTMARKETING }\end{array}$ & $85 \%$ & $64 \%$ & Not tested & $88 \%$ & $81 \%[1]$ \\
\hline $\begin{array}{l}\text { exp DRUG } \\
\text { HYPERSENSITIVITY OR exp } \\
\text { DRUG TOXICITY OR exp } \\
\text { PRODUCT SURVEILLANCE, } \\
\text { POSTMARKETING }\end{array}$ & $4 \%$ & $0 \%$ & Not tested & $10 \%$ & $7 \%$ \\
\hline $\begin{array}{l}\text { text word 'adverse' in the title } \\
\text { or abstract }\end{array}$ & $16 \%[2]$ & $29 \%[2]$ & $10 \%$ & $54 \%$ & $50 \%$ \\
\hline $\begin{array}{l}\text { synonyms of 'adverse effects' } \\
\text { in the title }\end{array}$ & $52 \%$ & $7 \%$ & Not tested & $18 \%$ & $19 \%$ \\
\hline $\begin{array}{l}\text { synonyms of 'adverse effects' } \\
\text { in the abstract }\end{array}$ & $45 \%[2]$ & $36 \%[2]$ & Not tested & $87 \%$ & $89 \%$ \\
\hline $\begin{array}{l}\text { synonyms of 'adverse effects' } \\
\text { in the title Or abstract }\end{array}$ & $63 \%[2]$ & $36 \%[2]$ & Not tested & $75 \%$ & $75 \%$ \\
\hline
\end{tabular}

[I] the subheadings 'adverse drug reaction', drug toxicity' and ' complication' were used in EMBASE as nearest equivalent to 'adverse effects', 'toxicity' and 'complications'. [2] searches in DARE and CDSR were limited to sections of the structured abstracts.

adverse effect, such as those examined in this study, are not indexed with adverse effect terms.

The variation in reported sensitivities from the different case studies may reflect their different inclusion criteria. Derry et al [3] limited their analysis to RCTs whereas Badgett et al [2] and Golder et al [4] included all types of primary studies. RCTs in particular, may not have adverse effects as the main outcome of the study and, therefore, may not contain this information in the title or abstract. This in turn means there is less information for an indexer to identify and index.
Derry et al [3] and Badgett et al [2] did not measure precision. The precision of the search strategies reported in Golder et al [4] (0\% to $9 \%)$ and the precision of the searches in CDSR in this study ( $0 \%$ to $3 \%$ ) are similar. However, the precision of the searches in DARE are higher in this study. For example, searching with 'floating' subheadings gave a precision of $16 \%$ in this study compared with just 3\% in Golder et al [4]. Searching for text words in the title and abstract gave $18 \%$ precision in this case study and 4\% in Golder et al [4]. The largest discrepancy was seen when searching with the MeSH term Exp DRUG TOXICITY where $67 \%$ precision was achieved in this 
study (although with a very low sensitivity at 1\%) compared to a precision of just $8 \%$ in Golder et al [4] (and sensitivity of 9\%). This higher precision in DARE may reflect the relative size of DARE and MEDLINE. 5\% (256/4919) of the total number of records on DARE were relevant to this case study and it would, therefore, not have been possible to achieve such low precision in DARE as that achieved by Golder et al [4] in MEDLINE.

\section{Limitations of the study}

Our searches were limited to CDSR and DARE. Although these are excellent sources of systematic reviews of adverse effects, not all reviews reported as being systematic are contained in these databases: DARE has a strict quality inclusion criterion and CDSR contains only Cochrane Reviews. These databases are sources of systematic reviews that tend to be of higher methodological quality, which may reflect better reporting and hence better indexing.

The low number of systematic reviews of adverse effects on CDSR (14) precluded any useful analysis of the data, including comparisons to DARE and previous research. In addition, the usefulness of individual search terms was difficult to assess because of a low number of records.

The search terms tested in this study were predefined from previous research and were not obtained by objective methods [10]. However, the papers $(n=13)$ not retrieved by the searches used in this study did not reveal many additional relevant terms.

\section{Conclusion}

Searching major systematic reviews databases for systematic reviews of adverse effects proved more difficult than anticipated due to a lack of standard terminology used by the authors of reviews, inadequate indexing and the variations in the search interfaces of these databases.

Our research suggests that it will be even more difficult to conduct thorough searches for systematic reviews that report adverse effects as a secondary outcome even in resources devoted to systematic reviews such as DARE and CDSR. At present hand searching all records in DARE and CDSR seems to be the only way to ensure retrieval of all systematic reviews of adverse effects in these databases.

\section{Key messages}

Every systematic review with adverse effect(s) as a primary outcome should be indexed with appropriate term(s).

Authors of systematic reviews should use standardised terminology to make it explicit that they are reviewing adverse effects.
Database producers and indexers need to improve the consistency of their indexing of adverse effects.

The publishers of The Cochrane Library and the producers of DARE could increase the utility of these databases to users - the former by allowing searches to be limited to sections of the structured abstracts in both DARE and CDSR records, and the latter by introducing the facility to search DARE with 'floating' subheadings.

\section{Competing interests}

The author(s) declare that they have no competing interests.

\section{Authors' contributions}

SG participated in the conception and design of the study, carried out the searches, sifted the records for relevant reviews and carried out an evaluation of the searches and helped draft the manuscript. YL carried out sifting of records for relevant reviews and helped draft the manuscript. HM conceived the study, sifted the records for relevant reviews, and helped draft the manuscript. All authors read and approved the final manuscript.

\section{Acknowledgements}

We would like to thank Julie Glanville from the Centre for Reviews and Dissemination (CRD) for comments on an earlier draft of the manuscript.

\section{References}

I. Ernst E, Pittler MH: Assessment of therapeutic safety in systematic reviews: literature review. $B M J$ 200I, 323:546.

2. Badgett R, Chiquette E, Anagnostelis B, Mulrow C: Locating reports of serious adverse drug reactions. 7th Annual Cochrane Colloquium Abstracts Rome 1999. PB54 (poster)

3. Derry S, Loke YK, Aronson K: Incomplete evidence: the inadequacy of databases in tracing published adverse drug reactions in clinical trials. BMC Medical Research Methodology 200 I, I :7.

4. Golder S, Mclntosh HM, Duffy S, Glanville J: Developing efficient search strategies to identify reports of adverse effects in MEDLINE and EMBASE. In Health Info Libr J Volume 23. Issue I ; 2006:3-12.

5. Wieland S, Dickersin K: Selective exposure reporting and Medline indexing limited the search sensitivity for observational studies of adverse effects of oral contraceptives. Journal of Clinical Epidemiology 2005, 58:560-7.

6. Centre for Reviews and Dissemination (CRD): How are systematic reviews identified for inclusion on DARE? York: Centre for Reviews and Dissemination (CRD). 2006 [http:// www.york.ac.uk/inst/crd/faq2a.htm].

7. Centre for Reviews and Dissemination (CRD): What will a DARE abstract tell me?: York: Centre for Reviews and Dissemination (CRD). 2006 [http://www.york.ac.uk/inst/crd/faqll.htm].

8. Higgins JPT, Green S, editors: Guide to the contents of a protocol and review. In: Higgins JPT, Green S, editors. Cochrane Handbook for Systematic Reviews of Interventions 4.2.5 [updated May 2005]; Section 3.3. 2005 [http:// www.cochrane.dk/cochrane/handbook/hbook.htm].

9. National Library of Medicine: Chapter 4: indexing operation: Bethesda: National Library of Medicine. 2005 [http:// www.nlm.nih.gov/mesh/indman/chapter 4.html].

10. White VJ, Glanville JM, Lefebvre C, Sheldon TA: A statistical approach to designing search filters to find systematic reviews: objectivity enhances accuracy. Journal of Information Science 200I, 27:357-70. 


\section{Pre-publication history}

The pre-publication history for this paper can be accessed here:

http://www.biomedcentral.com/1471-2288/6/22/prepub

Publish with Bio Med Central and every scientist can read your work free of charge

"BioMed Central will be the most significant development for disseminating the results of biomedical research in our lifetime. " Sir Paul Nurse, Cancer Research UK

Your research papers will be:

- available free of charge to the entire biomedical community

- peer reviewed and published immediately upon acceptance

- cited in PubMed and archived on PubMed Central

- yours - you keep the copyright 